
\title{
25 Research Square \\ Effects of Different Vegetable Rotations on the Bacterial Community Structure and Tomato Growth in a Continuous Tomato Cropping Substrate
}

Li Jin

Gansu Agricultural University

Jihua Yu

Gansu Agricultural University https://orcid.org/0000-0001-5849-7636

Ning Jin

Gansu Agricultural University

Jianming Xie

Gansu Agricultural University

Yue Wu

Gansu Agricultural University

Guobin Zhang

Gansu Agricultural University

Zhi Feng

Gansu Agricultural University

Zhongqi Tang

Gansu Agricultural University

Zeci Liu

Gansu Agricultural University

Shilei Luo

Gansu Agricultural University

Jian Lyu ( $\sim$ lvjian@gsau.edu.cn )

Gansu Agricultural University

\section{Research Article}

Keywords: tomato, continuous tomato cropping, crop rotation, organic ecotype soilless culture substrate, bacterial community

Posted Date: February 24th, 2021

DOI: https://doi.org/10.21203/rs.3.rs-196685/v1 
License: (c) (i) This work is licensed under a Creative Commons Attribution 4.0 International License. Read Full License 


\section{Abstract}

Purpose In this study, MiSeq pyrosequencing was used to analyze the effects of different vegetable rotations on the bacterial diversity and community structure in a substrate that was used for continuous tomato cropping (CK).

Methods The vegetable rotations tested were cabbage/tomato (B), kidney bean/tomato (D), and celery/tomato (Q).

Results The results revealed that the substrate bacterial diversity and richness of each crop rotation were higher than those of CK. The highest bacterial diversity was found in the B substrate, followed by the $Q$ and $D$ substrates. Further comparison showed that the bacterial community structure of $Q$ substrate was significantly different to that of CK. Compared with the CK, the $\mathrm{Q}$ substrate had a significantly higher relative abundance of several dominant microflora, such as Acidobacteria, Chloroflexi, and Firmicutes. Additionally, the $\mathrm{Q}$ rotation significantly increased the abundance of beneficial bacteria, such as Actinobacteria_unclassified and Anaerolineaceae_unclassified. A redundancy analysis showed that Most dominant bacteria correlated positively with the substrate $\mathrm{pH}$, total $\mathrm{N}$, and alkali-hydrolyzable $\mathrm{N}$ but negatively with the available $\mathrm{P}$, available $\mathrm{K}$, total $\mathrm{P}$, total $\mathrm{K}$, and organic matter contents and substrate $\mathrm{EC}$. The substrates after crop rotation improved the growth and physiological condition of the subsequent tomato plants, among which those from the $\mathrm{Q}$ rotation performed the best.

Conclusion Therefore, celery rotation not only increased the richness and diversity of bacterial communities in the substrate but also significantly increased the richness of the beneficial bacterial communities, allowing better maintenance of the substrate microenvironment for the healthy growth of crops.

\section{Introduction}

Organic substrates are widely applied for plant cultivation in solar greenhouses in China, having been easily extended owing to their low costs, less strict requirements of the external environment, and simple cultivation management. However, the practice of continuous cropping eventually leads to deterioration of the physicochemical properties of the organic substrate, and aggravation of plant diseases and insect pests (Tian-lai 2005). Aside from the deterioration of the substrate, continuous monoculture can also increase the presence of harmful microorganisms, thereby destroying the microecological balance (Lyu et al. 2020). Studies have shown that substrate problems still lead to continuous cropping obstacles, and the high temperature and humidity in the greenhouse serve to aggravate the occurrence of crop diseases and insect pests. Therefore, the ability to reuse the substrate is the main problem restricting the development of soilless substrate cultivation (Li et al. 2001).

Because of its high potential yield, crop water productivity, and profitability, the tomato plant (Lycopersicon esculentum Mill., belonging to the family Solanaceae) has quickly become one of the major vegetables grown in solar greenhouses in arid regions of northwestern China (Qiu et al. 2013). With 
the increase in tomato planting areas in the region, the continuous cultivation of the crop is becoming increasingly prevalent (Zheng et al. 2020). It is well known that the continuous cropping of a single species causes plant growth retardation, serious diseases and insect pests, low crop productivity, soil degradation, and microecosystem imbalance (Liu et al. 2014; Zhou et al. 2011). The diversity and richness of soil microorganisms play a particularly important role in maintaining soil health and promoting crop growth (Zhou et al. 2017). In recent years, most studies on ways to overcome or alleviate the obstacles of continuous cropping have focused mainly on changes in the rhizosphere soil microorganisms after the continuous cultivation of crops (Qin et al. 2017; Xiong et al. 2015). However, there are few reports on the changes in the organic substrate quality and microorganisms caused by tomato monoculture for several years.

Crop rotation (e.g., leguminous crop diversification) is a strategy used for maintaining soil quality and crop productivity to the high degree that single cropping cannot (Tiemann et al. 2015). The differences in the amount and composition of root exudates due to rotation affect the physicochemical properties of soil and the microbial communities therein (el Zahar Haichar et al. 2008). Additionally, the different plant-microbe interactions also affect the soil microbial communities, leading to less soil-borne diseases (Navarro-Noya et al. 2013).

In this study, a high-throughput sequencing method was used to study the bacterial diversity and community structures in organic substrates that had first been used for continuous tomato cropping and then rotated for the cultivation of different vegetables (cabbage, kidney bean, and celery). We compared the continuous tomato cropping system with three different vegetable/tomato rotation systems to achieve the following objectives: (1) to determine the changes in the bacterial diversity and community structure in the continuous tomato cropping substrate; (2) to explore the relationship between the bacterial community and physicochemical characteristics of the substrate; and (3) to evaluate which vegetable is the best for tomato rotation.

\section{Materials And Methods}

\subsection{Experimental design}

The test substrate was an organic ecotype soilless culture substrate made up of a mixture of slag, spent mushroom, cow manure, chicken manure, and corn straw at the ratios of 13:5:5:2:14, respectively. From June 2012 to June 2018, the continuous cropping experiment was conducted in the solar greenhouse of "Zongzhai non cultivated land facility agricultural demonstration park" in Zongzhai Town, Suzhou District, Jiuquan City, Gansu Province, China (98 $20^{\prime} \sim 99^{\circ} 18^{\prime} \mathrm{E}, 39^{\circ} 10^{\prime} \sim 39^{\circ} 59^{\prime} \mathrm{N}$; a region with a typical continental climate, with an average sea level of $1360 \mathrm{~m}$, annual average temperature of $7.3^{\circ} \mathrm{C}$, annual average precipitation of $176 \mathrm{~mm}$, and annual sunshine hours of 3033-3316 h). The continuous cropping vegetable was tomato (Lycopersicon esculentum Mill.), which was planted twice a year. In China, the overwintering crop is generally raised in September, planted in October, collected in the first 10 days of February of the next year, and pulled on time in May of that same year. Summer and autumn 
crops are generally planted according to the overwintering schedule, with most being planted in June, harvested in the first 10 days of August, and planted on time again in October. The experimental tomato variety used was "Jingfan 501," a pink fruit of infinite growth type. The plants were spaced $45 \mathrm{~cm}$ apart, with a row spacing of $25 \mathrm{~cm}$ and 30 plants in each plot. After continuous cropping (with 12 crops planted over 6 years), the substrate (designated CK) would usually contain the following amounts of macronutrients: total potassium $(K)$ of $11.78 \mathrm{~g} \cdot \mathrm{kg}^{-1}$, total phosphorus $(\mathrm{P})$ of $1.31 \mathrm{~g} \cdot \mathrm{kg}^{-1}$, total nitrogen (N) of $0.51 \mathrm{~g} \cdot \mathrm{kg}^{-1}$, available $\mathrm{P}$ of $82.81 \mathrm{mg} \cdot \mathrm{kg}^{-1}$, available $\mathrm{K}$ of $63.17 \mathrm{mg} \cdot \mathrm{kg}^{-1}$, and alkali-hydrolyzable $\mathrm{N}$ of $907.67 \mathrm{mg} \cdot \mathrm{kg}^{-1}$. Moreover, it typically has an electrical conductivity (EC) of $1683.67 \mu \mathrm{S} \cdot \mathrm{cm}^{-1}$ and a pH of 6.37 .

The crop rotation experiments were conducted in a glass greenhouse at Gansu Agricultural University from August 2018 to March 2019. The rotation vegetables were cabbage (Brassica pekinensis Rupr.), kidney bean (Phaseolus vulgaris Linn.), and celery (Apium graveolens L.), designated B, D, and Q, respectively, and tomato continuous cropping was designated $\mathrm{CK}$. The CK substrate collected from the continuous tomato cropping experimental site was transported to the greenhouse at Gansu Agricultural University and placed into $19 \mathrm{~cm} \times 30 \mathrm{~cm}$ pots ( $5 \mathrm{~kg}$ of substrate for each pot). The various vegetable seedlings that had been raised in advance were individually subplanted into pots. The field management of each crop rotation experiment was consistent with the local conventional management measures. Each rotation experiment was carried out in triplicate.

\subsection{Substrate sampling}

The substrate samples were collected after the rotation plant seedlings had been pulled (March 2019). After removing the $0-5 \mathrm{~cm}$ surface substrate and gently shaking off the loose substrate around the root system, the substrate adhered to the root surface was brushed off for collection and immediately stored as the rhizosphere sample in an ice box. The rhizosphere samples were extracted in triplicate for each the four crop experiments (i.e., CK, B, D, and Q), totaling 12 samples.

\subsection{DNA extraction}

The total DNAs of the microorganisms in $0.5 \mathrm{~g}$ of rhizosphere sample were extracted using the EZNA® Soil DNA Kit (OMEGA, Bio-Tek, Norcross, GA, USA) according to the manufacturer's protocols. For each crop experiment, the solutions of DNA extracted from the triplicate samples were pooled.

\subsection{Quantitative PCR and Illumina MiSeq sequencing}

The purified DNA was used as a template for polymerase chain reaction (PCR) amplification of the 16S rDNA V3 + V4 region, using the primers $338 \mathrm{f}$ (5'- actcctacgggaggcagcag-3') and 806r (5'ggactachvggggtwtctaat-3') (Bokulich et al. 2013). The $25-\mu \mathrm{L}$ reaction system consisted of $12.5 \mu \mathrm{L}$ of Phusion Hot Start Flex 2x Master Mix, $2.5 \mu \mathrm{L}$ of forward primer, $2.5 \mu \mathrm{L}$ of reverse primer, $50 \mathrm{ng}$ of template DNA, and enough $\mathrm{ddH}_{2} \mathrm{O}$ to make up the final volume. The PCR was carried out on an $\mathrm{ABI}$ GeneAmp ${ }^{\circledR}$ system (Model 9700; Applied Biosystems, Foster City, CA, USA) with the following amplification conditions: predenaturation at $98^{\circ} \mathrm{C}$ for $30 \mathrm{~s}$, denaturation at $98^{\circ} \mathrm{C}$ for $10 \mathrm{~s}$, annealing at 
$54^{\circ} \mathrm{C}$ for $30 \mathrm{~s}$, extension at $72^{\circ} \mathrm{C}$ for $45 \mathrm{~s}$, for a total of 35 cycles, and a final extension at $72^{\circ} \mathrm{C}$ for 10 min. Each sample was prepared in triplicate, and the final amplification product was detected by $1 \%$ agarose gel electrophoresis. The PCR products were then purified using an AxyPrep DNA gel extraction kit (AxyGen Biosciences, Union City, CA, USA) and quantified using a Quant-iT PicoGreen dsDNA assay kit (Promega, Madison, WI, USA) on a quantitative fluorescence system (QuantiFluor, Promega). (The qualifying library concentration should be more than $2 \mathrm{nM}$.) After diluting the qualified online sequencing libraries (the index sequence is not repeatable), they were mixed to the required sequencing amount according to the corresponding proportion. $\mathrm{NaOH}$ was then used to transform the molecules into a single chain for online sequencing with the MiSeq sequencer. For $2 \times 250$-bp double-terminal sequencing, the corresponding reagent to use is MiSeq Reagent Kit v2500 cycles.

\subsection{Processing of the sequencing data}

The original MiSeq data were obtained as an image file. After base calling, a result file stored in fastq format was obtained and then quality filtered using QIIME (version 1.17). PEAR (version 0.9.6) (Zhang et al. 2014) and Vsearch (version 2.3.4) (Rognes et al. 2016) were used to splice the two terminal sequences of the original data and filter the chimeric sequence. The Vsearch algorithm clustered the sequences with a greater than $97 \%$ similarity. Then, the representative operational taxonomic unit (OTU) sequences obtained by the clustering analysis were compared with sequences on the Ribosomal Database Project (version 11.5) (Cole et al. 2009; Cole et al. 2014) and SILVA databases (Schloss et al. 2009) to obtain the species annotation results of all OTUs.

\subsection{Determination of the growth physiology of subsequent tomato plants}

\subsubsection{Determination of the growth index}

The plant height was determined as the height from the base of the root to the growth point of the plant. The leaf area was determined using an LI-3000C leaf area meter.

\subsubsection{Determination of physiological indices}

Photosynthetic parameters: From tomato plants at their peak, the leaves at the same leaf position (i.e., the second leaf under the first inflorescence) were selected to measure their net photosynthetic rate (Pn), transpiration rate $(\mathrm{Tr})$, stomatal conductance (Gs), and intercellular $\mathrm{CO}_{2}$ concentration (Ci), using a CIRAS2(PP-System, UK) portable photosynthetic analyzer on the morning of a clear day. The values for each leaf were read three times.

Fluorescence parameters: Well-growing functional leaves were selected for determination of the levels of initial fluorescence (Fo), maximum fluorescence ( $\mathrm{Fm}$ ), variable fluorescence (Fv), photochemical quenching of photosystem II ( $\mathrm{q}^{\mathrm{P}}$ ), and non-photochemical quenching (NPQ), using an FMS-2 portable pulse-modulated fluorescence meter (Hansatech Instruments, Norfolk, UK).

\subsection{Statistical analysis}


Statistical analysis of the data was performed using the R packages Stats and Vegan (version 2.3-5) (Oksanen et al. 2016). Alpha-diversity indices (Shannon index, Simpson index, Chao1 index, and number of observed species) were calculated using QIIME (alpha_diversity.py). For the beta-diversity analysis, cluster analysis (sample clustering by Bray-Curtis distance) was used to show the similarity between samples, and weighted UniFrac distance measurement (based on system development structure) was used to generate a principal coordinate analysis ( $\mathrm{PCOA})$ map to further evaluate the similarity between community members of the samples (Lozupone et al. 2006). Redundancy analysis (RDA) was performed with the RDA function in the Vegan package in R (version 2.1.3) to study the effects of the physicochemical properties of the substrate on the composition of the rhizosphere fungal community. The tomato growth physiological data were analyzed by one-way analysis of variance using Excel 2016 and SPSS 20.0. The significance level for differences in this study was set at $P<0.05$.

\section{Results}

\subsection{Effects of different vegetable rotations on the bacterial diversity in the continuous tomato cropping substrate}

The changes in bacterial alpha-diversity index values of the various rotation and continuous cropping rhizosphere samples are shown in Table 1. The number of observed species (3242.33) and Chao1 index (6031.56) were the highest for the B rhizosphere, indicating that it had the highest bacterial community diversity and richness. The $D$ rhizosphere had the highest Shannon index (10.58). Additionally, the order of the index values for the various experiments were $B>D>Q>C K$ for observed species, $D>B>Q>C K$ for the Shannon index, and $B>Q>D>C K$ for the Chao1 index. These results showed that all four cropping systems tested could improve the richness and diversity of bacteria in the CK substrate to a certain extent.

Table 1

Comparison of species numbers and 16S (bacterial) diversity indices observed in the different cropping systems

\begin{tabular}{|lllll|}
\hline $\begin{array}{l}\text { Cropping } \\
\text { system }\end{array}$ & Observed species & Shannon index & Simpson index & Chao1 index \\
\hline CK & $2839.33 \pm 112.34 \mathrm{~b}$ & $10.40 \pm 0.07 \mathrm{a}$ & $1.00 \pm 0.00 \mathrm{a}$ & $5129.79 \pm 364.28 \mathrm{a}$ \\
\hline $\mathrm{Q}$ & $2978.67 \pm 25.12 \mathrm{ab}$ & $10.46 \pm 0.02 \mathrm{a}$ & $1.00 \pm 0.00 \mathrm{a}$ & $5472.59 \pm 223.76 \mathrm{a}$ \\
\hline B & $3242.33 \pm 122.83 \mathrm{a}$ & $10.51 \pm 0.09 \mathrm{a}$ & $1.00 \pm 0.00 \mathrm{a}$ & $6031.56 \pm 339.80 \mathrm{a}$ \\
\hline D & $3051 \pm 133.63 \mathrm{ab}$ & $10.58 \pm 0.05 \mathrm{a}$ & $1.00 \pm 0.00 \mathrm{a}$ & $5373.15 \pm 449.44 \mathrm{a}$ \\
\hline
\end{tabular}

CK: continuous tomato cropping; Q: celery/tomato rotation; B: cabbage/tomato rotation; D: kidney bean/tomato rotation. Different lowercase letters in a row indicate that the differences are statistically significant $(P<0.05)$. 
Figure 1-A shows the beta-diversity of the bacterial communities in the each crop experiment substrates as obtained by hierarchical clustering analysis. As shown in the figure, the bacterial communities were divided into three cluster groups: groups $C K$ and $D$, group $Q$, and group $B$. These groupings indicated that the bacterial community structures of the $C K$ and $D$ rhizospheres were similar but vastly different from those of the other two rhizospheres, and the bacterial community structures of the $\mathrm{Q}$ and $\mathrm{B}$ rhizospheres were also different from each other. Additionally, the difference in the fungal community composition between the $B$ and CK rhizospheres was the greatest, and B was separated from those of the $D$ and $Q$ rhizospheres.

The UniFrac weighted PCoA based on OTU components also clearly showed the difference between the final CK substrate and those from the different vegetable rotations, where the two axes explained $36.08 \%$ and $17.65 \%$ of the total bacterial data, respectively. Additionally, the bacterial communities in the CK and $D$ rhizosphere samples ordinated closely together in $\mathrm{PCo} 2$ and were significantly separated from those of the $B$ and $Q$ rhizospheres. (Fig. 1-B).

\subsection{Effects of different vegetable rotations on the bacterial community composition in the continuous tomato cropping substrate}

Although the diversity of the bacterial community structures in the four samples was similar, the abundance was different. The abundance of the bacterial community structure under each crop experiment is shown at the phylum level in Fig. 2. In total, 32 bacterial phyla were observed. The common taxa in the substrate were Proteobacteria (37.04-34.09\%), Actinobacteria (20.75-19.68\%), Acidobacteria (13.54-6.97\%), Bacteria_unclassified (7.75-7.02\%), Chloroflexi (8.98\%6.29\%), Gemmatimonadetes (7.29-5.17\%), and Firmicutes (4.30-3.44\%). The abundance of Proteobacteria was the highest in the B rhizosphere, accounting for $37.04 \%$ of the total bacteria in that substrate, and was $8.65 \%$ higher than that in the control CK substrate, which had the lowest Proteobacteria richness. The abundance of Actinobacteria was the highest in the CK substrate, whereas it was decreased in all the rotation substrates, being the lowest in the B rhizosphere (5.16\% lower than that of CK). The Q rhizosphere had the highest abundance of Chloroflexi and Firmicutes, which were $24.55 \%$ and $20.79 \%$ higher than that in the CK rhizosphere, respectively.

Figure 3 shows the thermogram of the diversity and richness of the bacterial communities in the four different rhizosphere samples, as represented by color gradient and similarity, where the color change from blue to red indicates a change in relative abundance of the community from low to high. In each crop rhizosphere, the proportion of Bacteria_unclassified (7.75-7.02\%) was the highest, with the order according to crop experiment being $Q>B>C K>D$. The genus in the second highest proportion was Gemmatimonas (7.28-5.16\%), in the order CK > B > D > Q. Sphingomonas and Gemmatimonas were the main genera in the substrates for continuous cropping, at abundance values of $1.41 \%$ and $7.28 \%$, respectively. In the Q rhizosphere samples, Hyphomicrobium, Anaerolineaceae_unclassified, Actinobacteria_unclassified, and Chloroflexus occurred at an abundance of $2.08 \%, 2.27 \%, 2.51 \%$, and $1.73 \%$, respectively. In the B rhizosphere, Solirubrobacter and Bacillariophyta were dominant, occurring at 
an abundance of $2.36 \%$ and $5.86 \%$, respectively. In the D sample, Rhizobium and Saccharibacteria_ genera_ incertae_sedis were dominant, being present at an abundance of $1.64 \%$ and $2.45 \%$, respectively.

\subsection{Effects of environmental factors on the distribution of bacteria and fungi in the continuous tomato cropping substrate}

The effects of environmental factors (i.e., physicochemical properties of the substrate) on the variation of the rich genera were determined by RDA. The RDA plot clearly shows the relationship between the physicochemical properties of the substrate and the bacterial community structure (Fig. 4), where the effects of the substrate properties on the indicated bacteria are reflected by the direction and length of the arrows. The position of the projection point of the sample on the arrow represents the approximate value of the factor in the corresponding sample. The first two axes of the RDA accounted for $80.64 \%$ and $7.33 \%$ of the total variation in the data. The results showed that the bacterial abundance in the B substrate was related to the available $\mathrm{P}$ content and the substrate EC. In the D substrate, the abundance of Bacillariophyta, Gemmatimonas, and Solirubrobacter was related to the contents of available P, available $\mathrm{K}$, total $\mathrm{P}$, total $\mathrm{K}$, and organic matter as well as the $\mathrm{EC}$. At the same time, the bacterial communities of the CK and Q substrates formed a separate group, such as Actinobacteria_unclassified, Gp6 and Gp16, Gaiella, etc., which were related to a higher $\mathrm{pH}$ value and total $\mathrm{N}$ and alkali-hydrolyzable nitrogen contents.

\subsection{Effects of the different vegetable rotations on tomato growth}

The height, stem diameter, and leaf area of the tomato plants grown under the various cropping systems were similar; that is, they all increased with the growth and development of the plants (Fig. 5). At the beginning of flowering, the height of the $Q$ plant was the highest, being significantly higher than that of the CK plant (by $0.44 \%$ ). By contrast, there was no significant difference in height between the $B, D$, and CK plants at this stage. At the early fruiting stage, the $D$ plant was significantly taller than the CK plant (by $0.46 \%$ ). By contrast, there was no significant difference in height between the $B$ and $Q$ plants. With the extension of the growth period, the height of the B plant increased the most obviously and reached its maximum value in the last two periods. Although there was no significant difference in stem diameter among the plants from all four cropping systems during the growth period, the value for each vegetable rotation was higher than that for $\mathrm{CK}$. There was also no significant difference in the leaf area among the plants from all four cropping systems at the beginning of flowering, with that of the B plant being the smallest. With the extension of the growth period, the leaf area of the B plant increased gradually, reaching its maximum in each subsequent growth period. The leaf area of the $D$ plant was significantly larger (by 15.42\%) than that of the CK plant at the early fruiting stage, but the difference between the two plants was no longer significant at the peak and late fruiting stages. The leaf area of the $Q$ plant was not significantly different from that of the CK plant, albeit it was still larger. 


\subsection{Effects of the different vegetable rotations on photosynthetic parameters of the tomato leaves}

Table 2

Effects of different vegetable rotations on light and photosynthetic parameters of the tomato leaves

\begin{tabular}{|lllll|}
\hline Cropping system & Ci & Tr & Gs & Pn \\
\cline { 2 - 5 } & $\left(\mu \mathrm{mol} \cdot \mathrm{mol}^{-1}\right)$ & $\left(\mathrm{mmol} \cdot \mathrm{m}^{-2} \cdot \mathrm{s}^{-1}\right)$ & $\left(\mathrm{mol} \cdot \mathrm{m}^{-2} \cdot \mathrm{s}^{-1}\right)$ & $\left(\mu \mathrm{mol} \cdot \mathrm{m}^{-2} \cdot \mathrm{s}^{-1}\right)$ \\
\hline CK & $377.5 \pm 8.53 \mathrm{~b}$ & $4.08 \pm 0.28 \mathrm{~b}$ & $322 \pm 29.17 \mathrm{~b}$ & $5.53 \pm 0.44 \mathrm{~b}$ \\
\hline Q & $421.5 \pm 11.42 \mathrm{a}$ & $6.65 \pm 0.36 \mathrm{a}$ & $531.5 \pm 19.4 \mathrm{a}$ & $8.21 \pm 0.59 \mathrm{a}$ \\
\hline B & $381.5 \pm 4.84 \mathrm{~b}$ & $6.9 \pm 0.59 \mathrm{a}$ & $485.75 \pm 18.87 \mathrm{a}$ & $7.25 \pm 0.55 \mathrm{ab}$ \\
\hline D & $398.5 \pm 4.52 \mathrm{ab}$ & $6.4 \pm 0.15 \mathrm{a}$ & $524.25 \pm 24.85 \mathrm{a}$ & $6.1 \pm 0.66 \mathrm{~b}$ \\
\hline
\end{tabular}

CK: continuous tomato cropping; Q: celery/tomato rotation; B: cabbage/tomato rotation; D: kidney bean/tomato rotation; $\mathrm{Ci}$ : intercellular $\mathrm{CO}_{2}$ concentration; $\mathrm{Tr}$ : transpiration rate; $\mathrm{Gs}$ : stomatal conductance; Pn: net photosynthetic rate. Different lowercase letters in a row indicate that the differences are statistically significant $(P<0.05)$.

The effects of different vegetable rotations on the light and photosynthetic parameters of the tomato leaves are shown in Table 2. Overall, the light and photosynthetic parameters of the leaves from tomato plants grown in the three crop rotation systems were higher than those of the leaves from the CK system. The Ci of the Q leaves was significantly higher (by 38.15\%) than that of the CK leaves. The Tr and GS of the $B$ and D leaves were significantly higher than those of the CK leaves (Tr increases of $62.99 \%(Q)$, $69.12 \%$ (B), and $58.86 \%$ (D) and Gs increases of $65.06 \%$ (Q), 50.85\% (B), and $62.81 \%$ (D), respectively). Compared with the $\mathrm{Pn}$ of the CK leaves, that of the Q leaves was significantly higher (by $48.46 \%$ ), whereas there was no significant difference between the B, D, and CK leaves for this parameter.

\subsection{Effects of the different vegetable rotations on the fluorescence parameters of the tomato leaves}

As shown in Table 3, there was no significant difference in Fv/Fm and Fv/Fo values between the leaves of each vegetable rotation and $C K$. The $D$ leaves had the highest $q^{P}$ value, which was significantly higher (by $4 \%$ ) than that of CK. However, there were no significant differences between the B, Q, and CK leaves for this parameter. The control CK leaves had the highest NPQ value, whereas the Q leaves had the lowest (47.83\% lower than CK), and the NPQ value of B leaf was second only to that of Q leaf, which was significantly lower than that of the control by $43.48 \%$. There was no significant difference in NPQ values between the $D$ and $C K$ leaves. 
Table 3

Effects of different vegetable rotations on the fluorescence parameters of tomato leaves

\begin{tabular}{|lllll|}
\hline Cropping system & Fv/Fm & Fv/Fo & q $^{\text {p }}$ & NPQ \\
\hline CK & $0.79 \pm 0.01 a$ & $3.28 \pm 0.19 a$ & $0.75 \pm 0.01 \mathrm{~b}$ & $0.23 \pm 0.02 \mathrm{a}$ \\
\hline Q & $0.82 \pm 0.02 \mathrm{a}$ & $3.81 \pm 0.29 \mathrm{a}$ & $0.76 \pm 0.02 \mathrm{ab}$ & $0.12 \pm 0.01 \mathrm{~b}$ \\
\hline B & $0.81 \pm 0.01 \mathrm{a}$ & $3.72 \pm 0.47 \mathrm{a}$ & $0.77 \pm 0.01 \mathrm{ab}$ & $0.13 \pm 0.01 \mathrm{~b}$ \\
\hline D & $0.8 \pm 0.01 \mathrm{a}$ & $3.42 \pm 0.3 \mathrm{a}$ & $0.78 \pm 0.003 \mathrm{a}$ & $0.16 \pm 0.02 \mathrm{ab}$ \\
\hline
\end{tabular}

CK: continuous tomato cropping; Q: celery/tomato rotation; B: cabbage/tomato rotation; D: kidney bean/tomato rotation; Fv: variable fluorescence; Fm: maximum fluorescence; Fo: initial fluorescence; $q^{p}$ : level of photochemical quenching of photosystem II; NPQ: non-photochemical quenching. Different lowercase letters in a row indicate that the differences are statistically significant $(P<0.05)$.

\section{Discussion}

Bacteria are the major drivers of nearly all biogeochemical cycles in terrestrial ecosystems and participate in maintaining the health and productivity of soils in agricultural systems (Nacke et al. 2011). Shiomi et al. found that it is difficult for pathogenic bacteria to grow and reproduce in soil with a high bacterial diversity, owing to the generally antagonistic and competitive nature of bacteria (Shiomi et al. 1999). Studies have shown that crop rotation provides an abundant diversity of bacterial groups in soil and that the most diverse rotations (four different crops) also have the most diverse and active soil microbiota. Therefore, the negative effects of agricultural intensification can be mitigated by designing more specific and diverse crop rotations (D'Acunto et al. 2018). It has also been shown that the structure and diversity indices of the soil bacterial community do not change significantly under rotation (Jiang et al. 2011). In our study, bacterial alpha-diversity analysis revealed that the observed characteristics and Chao1 index of the $B$ (cabbage) rotation were the highest; that is, the $B$ rotation had the highest bacterial richness, followed by the $Q$ (celery) rotation. Meanwhile, the $D$ (kidney bean) rotation had the highest Shannon index, indicating the substrate of this crop rotation had the highest bacterial diversity. The diversity and richness of the bacterial community in the control CK (continuous tomato cropping) substrate were lower than those in the crop rotation substrates. Therefore, compared with continuous monoculture, rotation can improve the diversity and richness of soil bacterial communities, as has been reported in many studies (González-Chávez et al. 2010; Postma-Blaauw et al. 2010; Venter et al. 2016).

Among the soil microorganisms, bacteria generally account for the highest proportion, and changes in their community structure are affected by the planted crops. Different crop species have different soil microbial environments as well as different compositions and abundance of bacterial community structures (Ghimire et al. 2014; WEN et al. 2016). In this study, bacterial community hierarchical clustering and PCoA2 analyses showed that the control CK substrate was significantly separated from substrates 
used in the rotations of cabbage and celery, that is to say, there were significant differences in bacterial community structure between continuous cropping substrate and substrate after rotation of cabbage and celery.

At the phylum level, the dominant group in most bacterial communities is Proteobacteria, which is the main phylum of heterotrophic gram-negative bacteria in various ecosystems, including soil, the plant leaf surface, the atmosphere, sea water, and freshwater. These bacteria can induce symbiotic $\mathrm{N}$ fixation with plants. The phylum contains not only pathogens of animals and plants but also beneficial bacteria that can inhibit pathogenic bacteria (Duangmal et al. 2014; Emmert and Handelsman 1999). In this study, the abundance of Proteus in all three vegetable rotation substrates was higher than that in the CK substrate. This result is supported by the fact that rotation can change soil nutrients by secreting different root substances, thus increasing the abundance of Proteus. (Fierer et al. 2007). Additionally, compared with continuous tomato cropping, celery rotation significantly increased the abundance of the Acidobacteria, Chloroflexus, and Firmicutes. These bacterial communities are important components of soil microbiotas and play important roles in promoting the healthy growth of plants, circulating soil material, and building a stable ecological environment (Lee et al. 2016). According to the bacterial thermogram of this study, the dominant bacteria in the rotation substrates belonged to the original control CK substrate, and the similarity was very high. The abundance of Sphingomonas and Gemmatimonas in the CK substrate was slightly higher than that in the rotation substrates. This result is corroborated by the study of Li et al. (LI et al. 2018). In the rotation substrates (B, D, Q), the abundance of actinomycetes, rhizobia, and unclassified anaeromycetes was significantly increased, with the highest abundance of actinomycetes and unclassified anaeromycetes observed in the $\mathrm{Q}$ substrate. Actinomycetes are a group of bacteria that can effectively inhibit the activity of soil-borne pathogens (Mendes et al. 2011), and their abundance in soil can be increased by rotation ( $\mathrm{Li}$ et al. 2017). Anaerobes, which are key groups in aquatic sediments and wetland soils (Huang et al. 2019; Williams 2015), are closely related to the degradation of organic carbon and organic compounds (Wang et al. 2018; Zhu et al. 2018).

The RDA plot (Fig. 4) revealed significant differences in the bacterial communities among all four rhizosphere samples. The available $\mathrm{P}$, total $\mathrm{N}$, total $\mathrm{K}$, organic matter, $\mathrm{EC}$, and $\mathrm{pH}$ were the main factors affecting the soil bacterial community structure. This may be because rotation, as a common agricultural cropping system, allows root exudates and crop residues of several crops to accumulate in the substrate, which enriches the nutrients available to the microorganisms and subsequently affects the bacterial community structure and diversity in the soil (Lange et al. 2015). Crop rotation can change the ecological environment of soil microorganisms because of the different crop uptakes of soil nutrients, water, and root exudates. In this study, the fast-growing leafy cabbage (B), the $\mathrm{N}$-fixing legume kidney bean (D), and the strongly allelopathic celery (Q) were selected for crop rotation in the substrate used for continuous tomato cropping. The results showed that the crop rotation had obvious effects on the bacterial community in the tomato continuous cropping substrate. It has previously been shown that the relative abundance of the dominant bacterial genera in soil after rotation was positively correlated with the soil acidity and alkalinity, available $\mathrm{K}$ content, and $\mathrm{C} / \mathrm{N}$ ratio, and negatively correlated with the available $\mathrm{P}$ content (Zhao et al. 2014). In our study, it was also found that Gp6 and Gp16, unclassified actinomycetes, 
and unclassified bacteria were positively correlated with the substrate $\mathrm{pH}$, total $\mathrm{N}$, and alkali-hydrolyzable $\mathrm{N}$ but negatively correlated with the available $\mathrm{P}$, available $\mathrm{K}$, total $\mathrm{P}$, total $\mathrm{K}$, and organic matter contents and the EC.

In this study, the physicochemical properties and microbial flora of the CK substrate were changed by the rotation of different vegetables in the previous crop cycle, thus affecting the growth physiology of subsequent tomato plants. It was found that the height, stem diameter, and leaf area of subsequent tomato plants were basically the same throughout the growth period, and the changes among the different crop rotations were not significant. However, these three parameters were higher overall for the tomato plants grown under each crop rotation system than for those grown under the continuous cropping system. In the study of Calonego et al. (Calonego and Rosolem 2010), it was also shown that Soybean grows more vigorously under rotation conditions.

Photosynthetic fluorescence is one of the most sensitive physiological processes affected when plants are subjected to external stress. Stress-induced damage of the photosynthetic structure hinders photosynthetic electron transport and reduces photosystem activity, thereby affecting photosynthetic carbon assimilation in the plant (Qi et al. 2011). With the increase in continuous cropping years, this cropping system leads to decreases in the root vigor, chlorophyll content, superoxide dismutase activity, $\mathrm{Pn}, \mathrm{Gs}, \mathrm{Tr}$, yield, and biomass of tomato plants, whereas crop rotation could largely alleviate these harmful effects (Fu et al. 2017; Suhui et al. 2016). In our study, the photosynthetic and fluorescence parameters of tomato plants grown under the continuous cropping system were worse than those grown under each rotation system, where the celery rotation significantly increased the $\mathrm{Ci}, \mathrm{Tr}, \mathrm{Gs}$, and $\mathrm{Pn}$ of subsequent tomato leaves, and the cabbage and bean rotations significantly increased the Tr and Gs of subsequent tomato leaves relative to the values for the control CK group. Bean rotation significantly increased the $\mathrm{q}^{\mathrm{P}}$ of subsequent tomato leaves, whereas celery rotation significantly decreased the NPQ of subsequent tomatoes. By contrast, celery rotation was more beneficial for improving the photosynthetic and fluorescence physiology of subsequent tomatoes. Ahmad et al. (Ahmad et al. 2013) also showed that the intercropping of garlic could significantly increase the chlorophyll content, $\mathrm{Pn}$, and antioxidant enzyme activity of pepper, and the allelochemicals of garlic played a crucial role in improving the physiological properties and biochemical reactions of pepper. Celery, used in our study, also belongs to a class of vegetables with strong allergens, which again proves the importance of allergens in alleviating the substrate disorders caused by continuous cropping.

\section{Conclusion}

In this experiment, the effects of rotation of cabbage, kidney bean and celery on bacterial diversity, community structure of continuous tomato cropping rhizosphere and growth of subsequent tomato were studied. All four cropping systems tested could improve the richness and diversity of bacteria in the continuous cropping substrate to a certain extent. The highest bacterial diversity was found in the cabbage substrate, followed by the celery rotation and kidney bean rotation substrates. Further comparison showed that the substrate bacterial community structure of kidney bean rotation was similar 
to that of continuous cropping of, whereas those of cabbage rotation and celery rotation (especially celery rotation) were significantly different to that of continuous cropping of. Additionally, the celery rotation significantly increased the abundance of beneficial bacteria, such as Actinobacteria_unclassified and Anaerolineaceae_unclassified. And the rotation of celery can better improve the growth and physiological condition of tomato. Therefore, celery rotation can better maintenance the substrate microenvironment for the healthy growth of crops and promotion of the growth and development of subsequent tomatoes and providing a more effective way to avoid continuous tomato cropping in greenhouses.

\section{Declarations}

This research was supported by National key R \& D plan (2018YFD0201205), Special project of central government guiding local science and technology development (ZCYD-2020-5-2), Special project of national modern agricultural industrial system (CARS-23-C-07), Gansu people's livelihood science and technology project (20CX9NA099).

\section{Author information}

Affiliations

\section{College of Horticulture, Gansu Agricultural University, Lanzhou, China}

Li Jin, Jian Lyu, Ning Jin, Jianming Xie, Yue Wu, Guobin Zhang, Zhi Feng, Zhongqi Tang, Zeci Liu, Shilei Luo, Jihua Yu

Corresponding author

Correspondence to Jian Lyu and Jihua Yu.

\section{Acknowledgements}

We sincerely thank the staff of theVegetable Technical Service Center of Suzhou District, Jiuquan City, for experimental field management.

\section{References}

Ahmad I, Cheng Z, Meng H, Liu T, Nan W, Khan M, Khan A (2013) Effect of intercropped garlic (Allium sativum) on chlorophyl. Pak J Bot 45:1889-1896

Bokulich NA et al (2013) Quality-filtering vastly improves diversity estimates from Illumina amplicon sequencing. Nature methods 10:57-59

Calonego JC, Rosolem CA (2010) Soybean root growth and yield in rotation with cover crops under chiseling and no-till European. Journal of Agronomy 33:242-249 
Cole JR et al (2009) The Ribosomal Database Project: improved alignments and new tools for rRNA analysis Nucleic acids research 37:D141-D145

Cole JR et al (2014) Ribosomal Database Project: data and tools for high throughput rRNA. analysis Nucleic acids research 42:D633-D642

D’Acunto L, Andrade JF, Poggio SL, Semmartin M (2018) Diversifying crop rotation increased metabolic soil diversity and activity of the microbial community Agriculture. Ecosystems Environment 257:159-164

Duangmal K, Poomthongdee N, Pathom-aree W, Takè A, Thamchaipenet A, Matsumoto A, Takahashi Y (2014) Allokutzneria oryzae sp. nov., isolated from rhizospheric soil of Oryza sativa. L International journal of systematic evolutionary microbiology 64:3559-3564

el Zahar Haichar F et al (2008) Plant host habitat and root exudates shape soil bacterial community structure. ISME J 2:1221-1230

Emmert EA, Handelsman J (1999) Biocontrol of plant disease: a (Gram-) positive perspective FEMS. Microbiology letters 171:1-9

Fierer N, Bradford MA, Jackson RB (2007) Toward an ecological classification of soil bacteria Ecology 88:1354-1364

Fu H, Zhang G, Zhang F, Sun Z, Geng G, Li T (2017) Effects of continuous tomato monoculture on soil microbial properties and enzyme activities in a solar greenhouse Sustainability 9:317

Ghimire R, Norton JB, Stahl PD, Norton U (2014) Soil microbial substrate properties and microbial community responses under irrigated organic and reduced-tillage crop and forage production systems. PloS one 9:e103901

González-Chávez MdCA, Aitkenhead-Peterson JA, Gentry TJ, Zuberer D, Hons F, Loeppert R (2010) Soil microbial community, C, N, and P responses to long-term tillage and crop rotation. Soil Tillage Res 106:285-293

Huang W, Chen X, Wang K, Chen J, Zheng B, Jiang X (2019) Comparison among the microbial communities in the lake, lake wetland, and estuary sediments of a plain. river network Microbiologyopen 8:e00644

Jiang X, Wright AL, Wang X, Liang F (2011) Tillage-induced changes in fungal and bacterial biomass associated with soil aggregates: a long-term field study in a subtropical rice soil in China. Appl Soil Ecol 48:168-173

Lange $M$ et al (2015) Plant diversity increases soil microbial activity and soil carbon storage. Nature communications 6:1-8 
Lee S, Yeo I-Y, Sadeghi AM, McCarty GW, Hively WD, Lang MW (2016) Impacts of watershed characteristics and crop rotations on winter cover crop nitrate-nitrogen uptake capacity within agricultural watersheds in the Chesapeake Bay region PloS one 11:e0157637

Li G-j, Xu Z-h, Benoit F (2001) The application of polyurethane ether foam (PUR) to soilless culture as an reusable and environmental sound substrate. Acta Agriculturae Zhejiangensis 13:61-66

Li T et al (2017) Changes in soil bacterial community structure as a result of incorporation of Brassica plants compared with continuous planting eggplant and chemical disinfection in greenhouses PloS one $12: e 0173923$

LI W-h LIU, Q-z, Peng C (2018) Effect of long-term continuous cropping of strawberry on soil bacterial community structure and diversity. Journal of integrative agriculture 17:2570-2582

Liu X, Zhang J, Gu T, Zhang W, Shen Q, Yin S, Qiu H (2014) Microbial community diversities and taxa abundances in soils along a seven-year gradient of potato monoculture using high throughput pyrosequencing approach. PloS one 9:e86610

Lozupone C, Hamady M, Knight R (2006) UniFrac-an online tool for comparing microbial community diversity in a phylogenetic context. BMC Bioinform 7:371

Lyu J et al (2020) Effects of Different Vegetable Rotations on Fungal Community Structure in Continuous Tomato Cropping Matrix in Greenhouse Frontiers in Microbiology 11

Mendes R et al (2011) Deciphering the rhizosphere microbiome for disease-suppressive. bacteria Science 332:1097-1100

Nacke $\mathrm{H}$ et al (2011) Pyrosequencing-based assessment of bacterial community structure along different management types in German forest and grassland soils. PloS one 6:e17000

Navarro-Noya YE et al (2013) Relative impacts of tillage, residue management and crop-rotation on soil bacterial communities in a semi-arid agroecosystem. Soil Biol Biochem 65:86-95

Oksanen J et al (2016) vegan: Community Ecology Package. R package version 2.4-3 Vienna: R Foundation for Statistical Computing[Google Scholar]

Postma-Blaauw MB, de Goede RGM, Bloem J, Faber JH, Brussaard L (2010) Soil biota community structure and abundance under agricultural intensification and extensification Ecology 91:460-473

Qi M, Liu Y, Zhou L, Li T, Fan Y, Zhang K (2011) Regulation of calcium on photosynthesis of tomato leaves under sub-high temperature stress. Scientia Agricultura Sinica 44:531-537

Qin S, Yeboah S, Xu X, Liu Y, Yu B (2017) Analysis on fungal diversity in rhizosphere soil of continuous cropping potato subjected to different furrow-ridge mulching managements. Frontiers in microbiology 
Qiu R, Song J, Du T, Kang S, Tong L, Chen R, Wu L (2013) Response of evapotranspiration and yield to planting density of solar greenhouse grown tomato in northwest China. Agric Water Manag 130:44-51

Rognes T, Flouri T, Nichols B, Quince C, Mahé F (2016) VSEARCH: a versatile open source tool. for metagenomics PeerJ 4:e2584

Schloss PD et al (2009) Introducing mothur: open-source, platform-independent, community-supported software for describing and comparing microbial communities. Appl Environ Microbiol 75:7537-7541

Shiomi Y, Nishiyama M, Onizuka T, Marumoto T (1999) Comparison of bacterial community structures in the rhizoplane of tomato plants grown in soils suppressive and conducive towards bacterial wilt. Appl Environ Microbiol 65:3996-4001

Suhui L, Jinqiang X, Hui W, Qingtao L, Zheng X (2016) Effect of Garlic Straw on Physical and Chemical Characteristics of Continuous Cropping Soil and Root Activity of Tomato in Solar Greenhouse Agricultural Science \& Technology 17

Tian-lai L (2005) Current Situation and Prospects of Green House Industry Development in China [J]. Journal of Shenyang Agricultural University 2:131-138

Tiemann L, Grandy A, Atkinson E, Marin-Spiotta E, McDaniel M (2015) Crop rotational diversity enhances belowground communities and functions in an agroecosystem. Ecology letters 18:761-771

Venter ZS, Jacobs K, Hawkins H-J (2016) The impact of crop rotation on soil microbial diversity: A metaanalysis. Pedobiologia 59:215-223

Wang C et al (2018) Bacterial communities and their predicted functions explain the sediment nitrogen changes along with submerged macrophyte restoration. Microbial ecology 76:625-636

WEN X-y, Dubinsky E, Yao W, Rong Y, Fu C (2016) Wheat, maize and sunflower cropping systems selectively influence bacteria community structure and diversity in their and succeeding crop's rhizosphere. Journal of integrative agriculture 15:1892-1902

Williams AS (2015) The dynamic interaction between microbial biodiversity, biogeochemical activity and sedimentary geomorphology in the Severn Estuary. Cardiff University

Xiong W et al (2015) Different continuous cropping spans significantly affect microbial community membership and structure in a vanilla-grown soil as revealed by deep pyrosequencing. Microbial ecology $70: 209-218$

Zhang J, Kobert K, Flouri T, Stamatakis A (2014) PEAR: a fast and accurate Illumina Paired-End reAd. mergeR Bioinformatics 30:614-620 
Zhao J, Zhang R, Xue C, Xun W, Sun L, Xu Y, Shen Q (2014) Pyrosequencing reveals contrasting soil bacterial diversity and community structure of two main winter wheat cropping systems in. China Microbial ecology 67:443-453

Zheng X, Wang Z, Zhu Y, Wang J, Liu B (2020) Effects of a microbial restoration substrate on plant growth and rhizosphere bacterial community in a continuous tomato cropping. greenhouse Scientific reports 10:1-11

Zhou X, Liu J, Wu F (2017) Soil microbial communities in cucumber monoculture and rotation systems and their feedback effects on cucumber seedling growth. Plant Soil 415:507-520

Zhou X, Yu G, Wu F (2011) Effects of intercropping cucumber with onion or garlic on soil enzyme activities, microbial communities and cucumber yield European. Journal of Soil Biology 47:279-287

Zhu P, Wang Y, Shi T, Zhang X, Huang G, Gong J (2018) Intertidal zonation affects diversity and functional potentials of bacteria in surface sediments: A case study of the Golden Bay mangrove. China Applied Soil Ecology 130:159-168

\section{Figures}
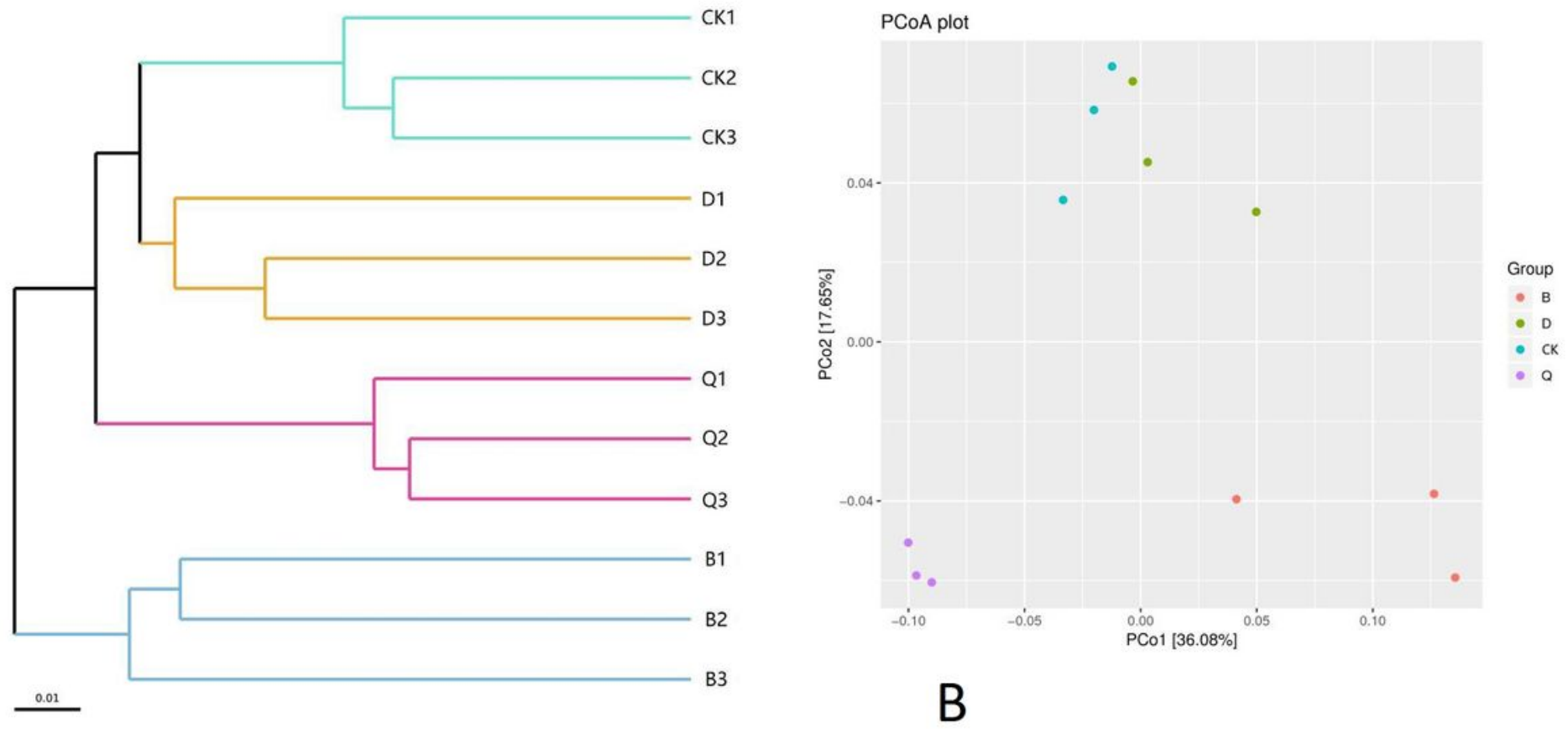

A

Figure 1

Hierarchical clustering (A) and principal coordinate analysis (B) of the bacterial communities in the continuous tomato cropping substrates based on different cropping systems. CK: continuous tomato 
cropping; B: cabbage/tomato rotation; D: kidney bean/tomato rotation; Q: celery/tomato rotation.

\section{Phylum}

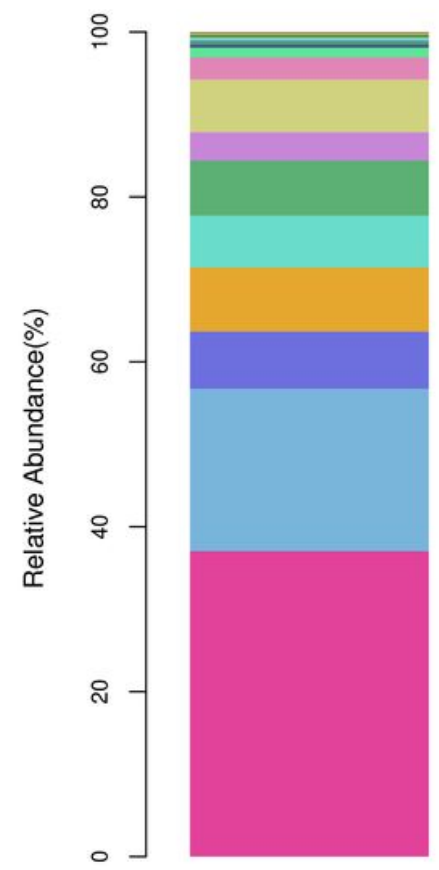

$\infty$

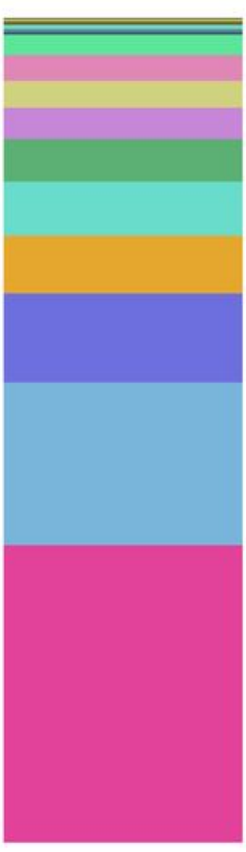

$\circ$

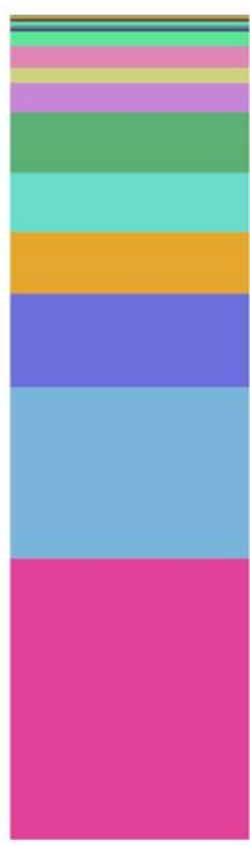

త

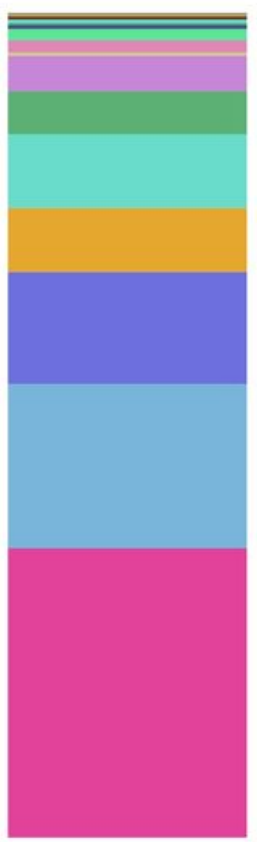

o
- Proteobacteria

- Actinobacteria

- Acidobacteria

- Bacteria_unclassified

- Chloroflexi

Gemmatimonadetes

E Firmicutes

- Cyanobacteria

- Bacteroidetes

- Candidatus_Saccharibacteria

- Planctomycetes

- Deinococcus-Thermus

- Nitrospirae

- Verrucomicrobia

- Chlamydiae

Latescibacteria

Parcubacteria

- Ignavibacteriae

- Fibrobacteres

- Armatimonadetes

- Others

\section{Figure 2}

Relative abundance of main bacterial genera (top 20) in continuous tomato cropping substrates under different cropping systems. CK: continuous tomato cropping; B: cabbage/tomato rotation; D: kidney bean/tomato rotation; Q: celery/tomato rotation. 


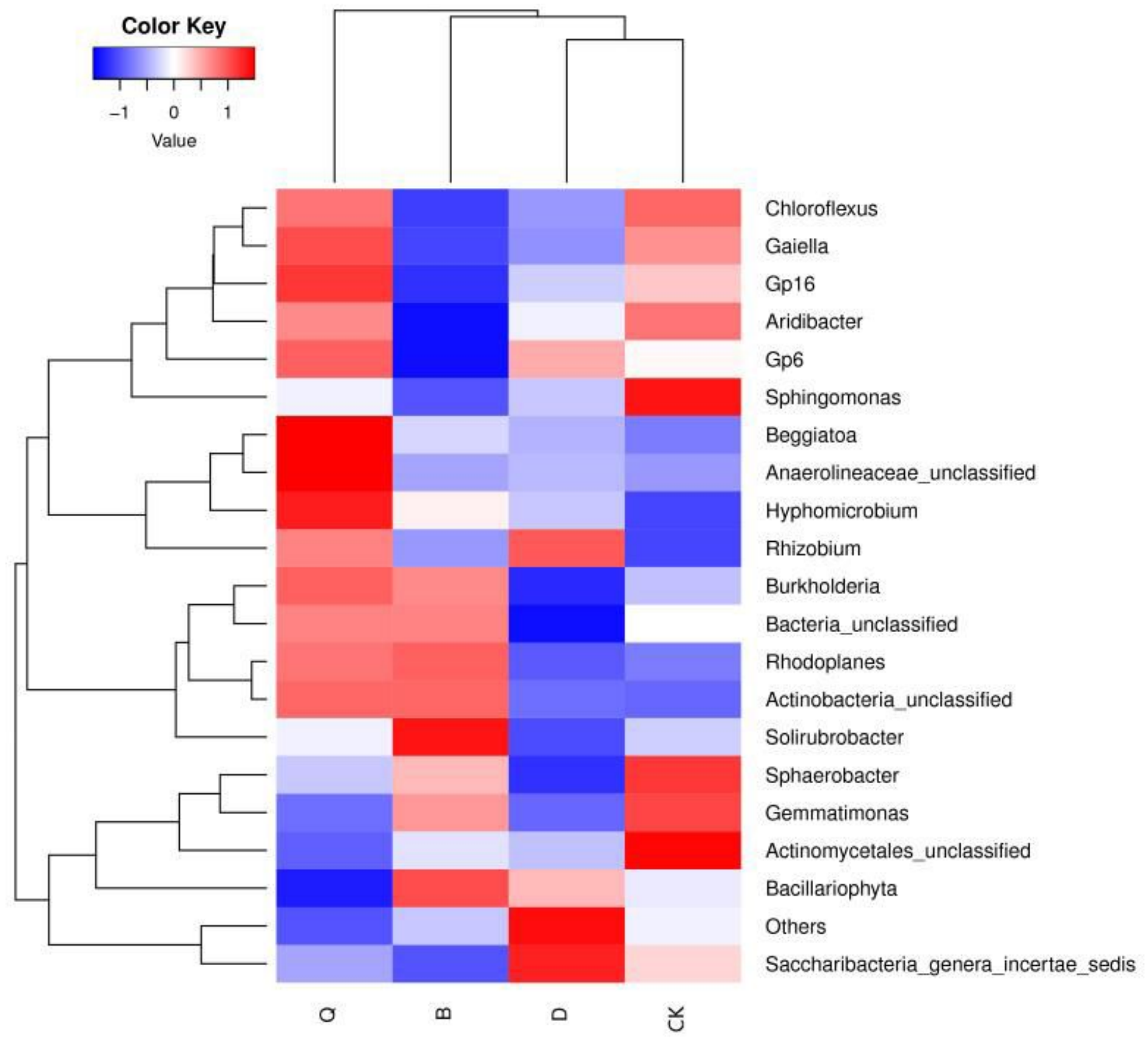

\section{Figure 3}

Thermogram of the changes in bacterial community diversity and richness in the continuous tomato cropping substrates under different cropping systems. CK: continuous tomato cropping; Q: celery/tomato rotation; B: cabbage/tomato rotation; D: kidney bean/tomato rotation. 


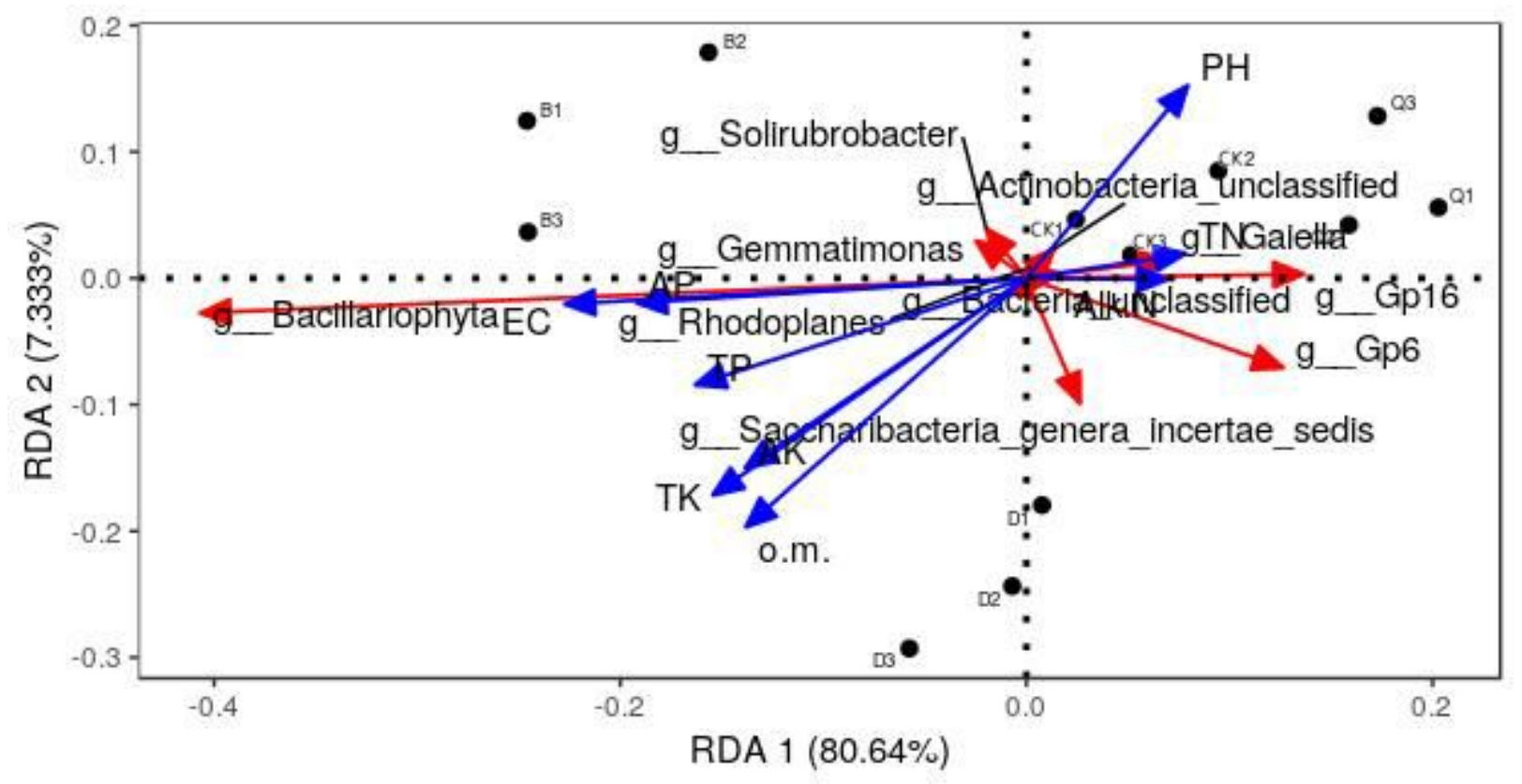

Figure 4

Redundancy analysis (RDA) of the effects of environmental factors on the bacterial community distribution in continuous cropping tomato substrates from different cropping systems. The dots represent the substrate sample, the blue arrows represent the physicochemical properties of the substrate, and the red arrows represent the substrate microorganism. An acute angle between the influencing factors (between the factors and the sample) indicates a positive correlation between the two factors, whereas an obtuse angle indicates a negative correlation. The longer the ray is, the greater is the effect of the factor. EC: electrical conductivity; $\mathrm{pH}$ : substrate $\mathrm{pH}$; ORM: organic matter; $\mathrm{TN}$ : total nitrogen; TP: total phosphorus; TK: total potassium; AP: available phosphorus; AK: available potassium; Alk N: alkalihydrolyzable nitrogen 

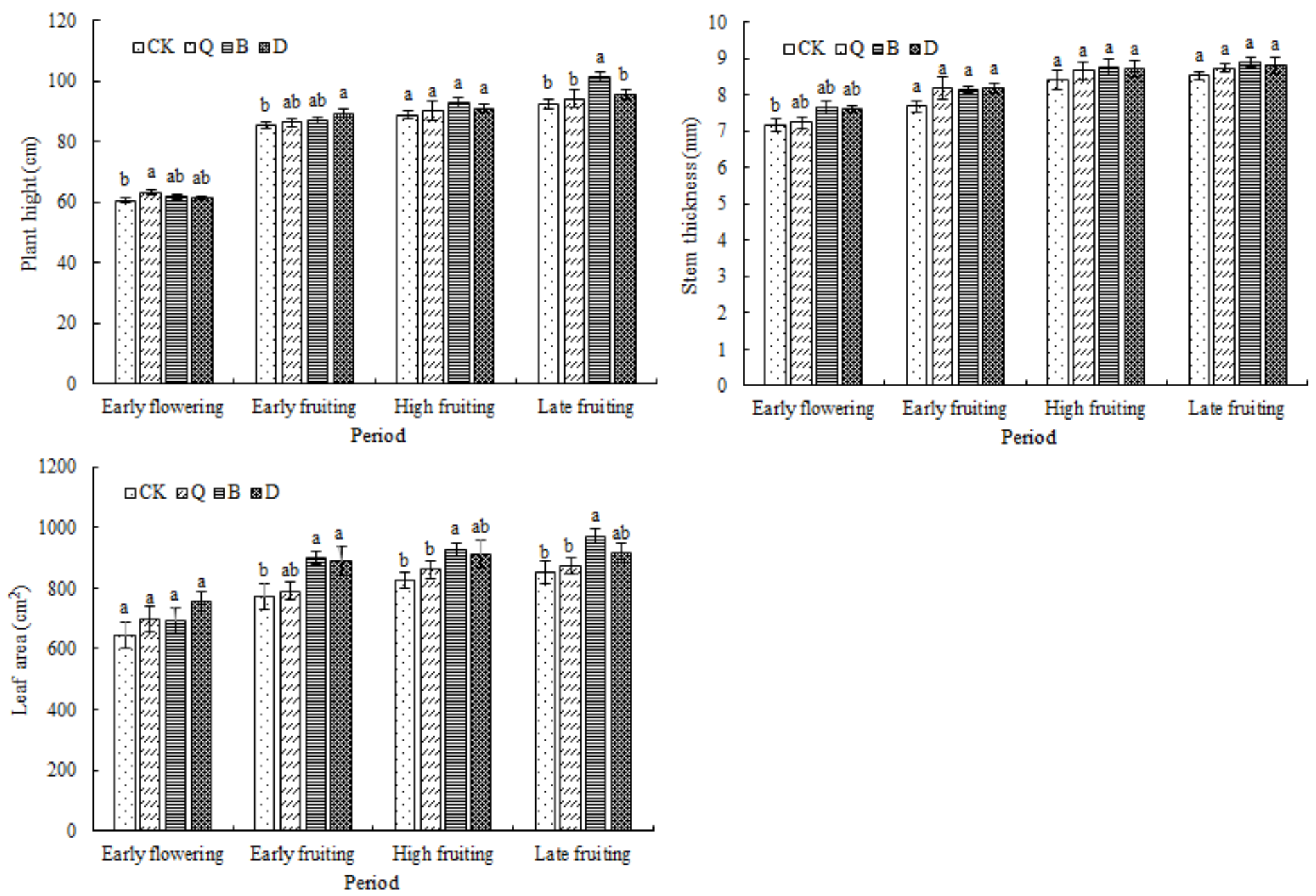

Figure 5

Effects of different vegetable rotations on tomato plant growth.CK: continuous tomato cropping; Q: celery/tomato rotation; B: cabbage/tomato rotation; $\mathrm{D}$ : kidney bean/tomato rotation.Different lowercase letters in a row indicate that the differences are statistically significant $(P<0.05)$. 